\title{
The synthesis of 2-arylquinoxaline derivatives
}

\author{
Jabbar Khalafy*, Bayram Parsa Habashi, Ahmad Poursattar Marjani and Peyman Najafi \\ Moghadam
}

Department of Chemistry, Faculty of Science, Urmia University, Urmia 57154, Iran

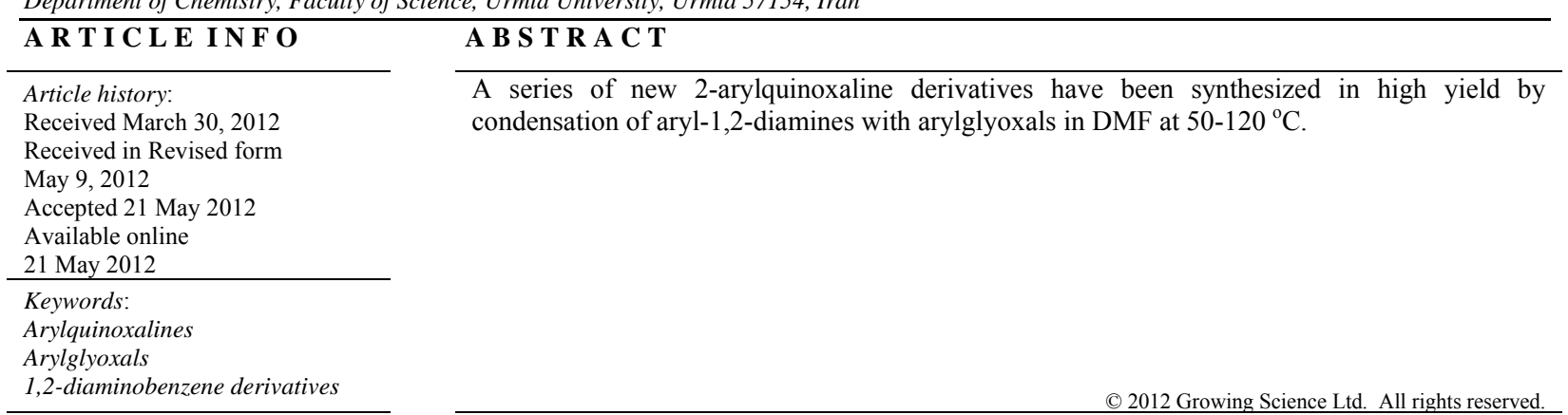

\section{Introduction}

Among the various classes of nitrogen-containing heterocyclic compounds, quinoxaline derivatives are an important component of pharmacologically active compounds. Quinoxaline derivatives have various pharamacological activities such as actinoleutin, hinomycin, and levomycin that are known to inhibit growth of gram positive bacteria and are active against various transplantable tumors. ${ }^{1-6}$ In addition quinoxaline derivatives are also associated with a wide spectrum of biological activities ranging from antibacterial, ${ }^{7-10}$ antifungal, ${ }^{7,11}$ antitubercular, ${ }^{7,12-14}$ analgesic ${ }^{9,15}$ and anti-inflammatory. ${ }^{15,16}$ We have reported the synthesis of quinoxaline derivatives from 2-bromo4-chloro-indanone ${ }^{17}$ and arylaminoisoxazol-5(2H)-ones. ${ }^{18}$ Here, we report a facile method for the synthesis of 2-arylquinoxalines in good to excellent yields by reaction of 1,2-diaminobenzene derivatives with various arylglyoxals in $\mathrm{DMF}$ at $50-120{ }^{\circ} \mathrm{C}$.

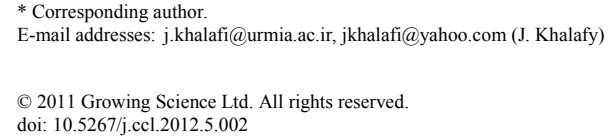




\section{Results and Discussion}

The arylglyoxals were prepared by oxidation of the corresponding acetophenones using $\mathrm{SeO}_{2}$ (Scheme 1). ${ }^{19}$

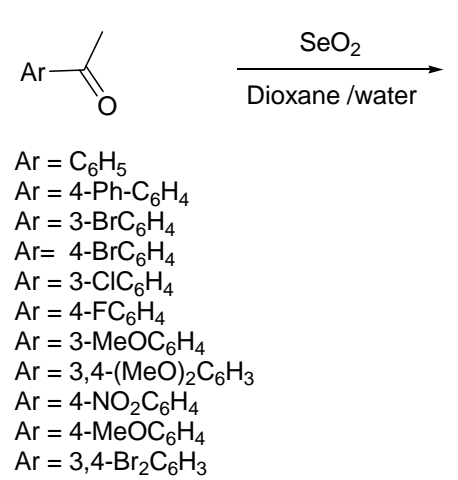<smiles>O=CC(=O)Br</smiles>

Scheme 1. Synthesis of arylglyoxals

The reaction of arylglyoxals (1) with aryl-1,2-diamines (2) in DMF at 50-120 ${ }^{\circ} \mathrm{C}$ afforded the corresponding 2-arylquinoxalines (3a-t) in 68-96\% yields (Scheme 2).

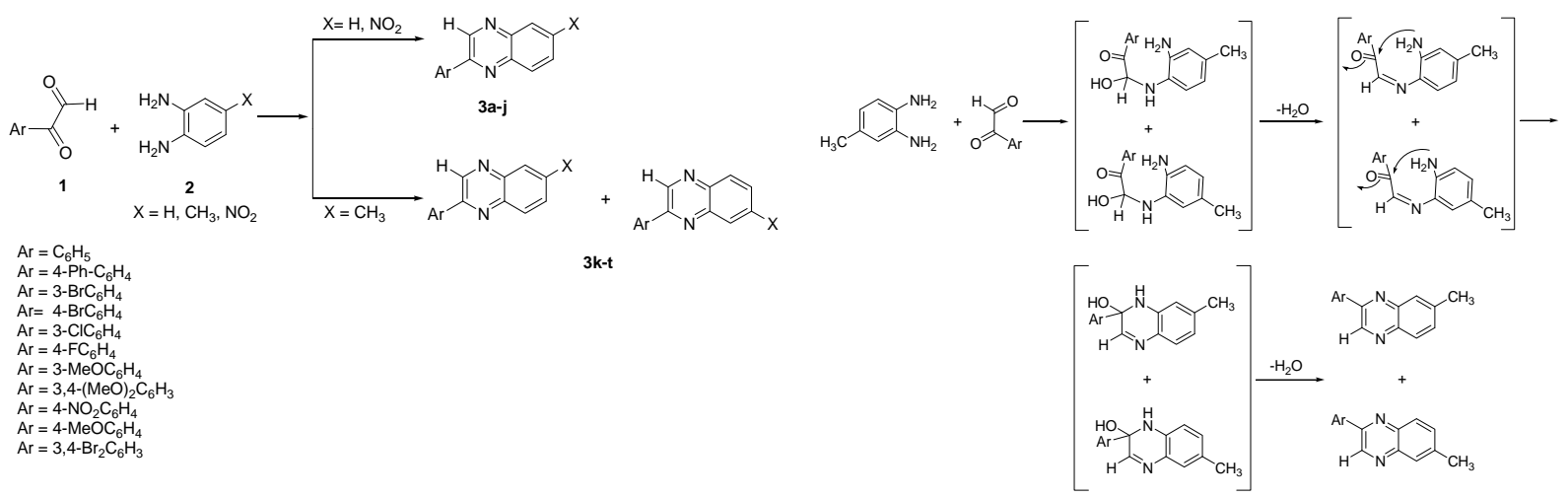

Scheme 2. Synthesis of 2-arylquinoxalines (3a-t) Scheme 3. Suggested mechanism for synthesis of 2-arylquinoxalines (3k-t)

The ${ }^{1} \mathrm{H}-\mathrm{NMR}$ spectra of compounds (3k-t) show two singlets in the region of $\delta=9.23-9.32 \mathrm{ppm}$, due to the formation of a mixture of two isomers. The proposed mechanism for the synthesis of 2arylquinoxalines (3k-t) is shown in Scheme 3. In case of $\mathbf{3 f - j}$ as the amino group in position 2 is more active than the amino group in position 4 (due to the resonance affect), therefore, it condenses with the formyl group of glyoxals in the first step to form a single product as shown in Scheme 4.

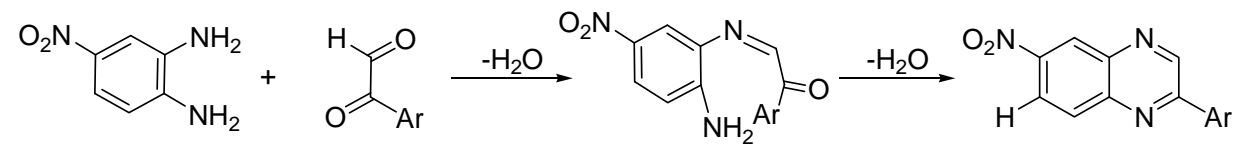

Scheme 4. Suggested mechanism for synthesis of 2-arylquinoxalines (3f-j)

It should be mentioned that repeating the reactions at temperatures higher than those mentioned in Table 1 will reduce the yields, due to decomposition. 
Table 1. Synthesis of 2-arylquinoxalines (3a-t)

\begin{tabular}{|c|c|c|c|c|}
\hline Entry & Product (3a-t) & Time (h) & $\begin{array}{l}\text { Reaction } \\
\text { Temperature }\left({ }^{\circ} \mathrm{C}\right) \\
\end{array}$ & Yield (\%) \\
\hline $\mathrm{a}$ & & 6 & 100 & 81 \\
\hline $\mathrm{b}$ & & 6 & 90 & 73 \\
\hline $\mathrm{c}$ & & 6 & 120 & 78 \\
\hline$d$ & & 6 & 100 & 79 \\
\hline e & & 5 & 120 & 92 \\
\hline$f$ & & 4 & 120 & 83 \\
\hline $\mathrm{g}$ & & 2 & 120 & 74 \\
\hline $\mathrm{h}$ & & 2 & 50 & 85 \\
\hline i & & 8 & 120 & 83 \\
\hline j & & 2 & 100 & 84 \\
\hline $\mathrm{k}$ & & 8 & 100 & 68 \\
\hline 1 & & 4 & 90 & 84 \\
\hline $\mathrm{m}$ & & 12 & 90 & 87 \\
\hline $\mathrm{n}$ & & 12 & 80 & 77 \\
\hline o & & 8 & 70 & 78 \\
\hline $\mathrm{p}$ & & 8 & 70 & 72 \\
\hline q & & 8 & 90 & 79 \\
\hline $\mathrm{r}$ & & 9 & 80 & 76 \\
\hline $\mathrm{s}$ & & 10 & 90 & 96 \\
\hline $\mathrm{t}$ & & 8 & 80 & 91 \\
\hline
\end{tabular}




\section{Conclusions}

The work reported herein provides a highly effective and simple one step method for the synthesis of new 2-arylquinoxalines, which may have pharmaceutical and biological applications.

\section{Acknowledgements}

The authors are grateful to the Urmia University for support of this work.

\section{Experimental}

General Procedure. ${ }^{1} \mathrm{H}$ and ${ }^{13} \mathrm{C}$ NMR spectra were recorded on a Bruker AM-300 spectrometer at $300 \mathrm{MHz}$ and $75.5 \mathrm{MHz}$, respectively. The spectra were measured in $\mathrm{CDCl}_{3}$ or DMSO- $\mathrm{d}_{6}$ using TMS as the internal standard. Infrared spectra were determined on a Thermo Nicolet (Nexus 670) FT-IR spectrometer, using $\mathrm{KBr}$ disks. Microanalyses were performed on a Carlo-Erba Analyzer 1104. Melting points were determined on a digital melting point apparatus (electrothermal) and remain uncorrected.

\section{General Procedure for the Synthesis of 2-Arylquinoxalines (3a-t)}

A mixture of arylglyoxal 1 (1 mmol), aryl-1,2-diamine $2(1 \mathrm{mmol})$, in DMF $(5 \mathrm{ml})$ were stirred at $50-120{ }^{\circ} \mathrm{C}$ for $2-12 \mathrm{~h}$. The completion of the reactions were monitored with thin-layer chromatography (TLC). After the appropriate time, water was added and the reaction mixture was stirred. The precipitate was then collected, and recrystallized from ethanol to afford pure 2arylquinoxalines (3a-t).

\section{2-(4-bromophenyl)quinoxaline (3a):}

Cream solid; mp: $135{ }^{\circ} \mathrm{C} .{ }^{1} \mathrm{H}$ NMR $\left(\mathrm{CDCl}_{3}\right)$ : 7.70-7.84 (m, 4H, ArH), 8.09-8.15 (m, 4H, ArH), 9.32 $(\mathrm{s}, 1 \mathrm{H}, \mathrm{ArH}) \mathrm{ppm} ;{ }^{13} \mathrm{C}$ NMR $\left(\mathrm{CDCl}_{3}\right): \delta 125.01,128.96,129.09,129.58,129.83,130.50,132.33$, 135.53, 141.53, 142.20,142.65, 150.58 ppm; FT-IR (KBr): v 3050, 2921, 1586, 1510, 1485, 1312, 1072, 1009, 828, $757 \mathrm{~cm}^{-1}$. Anal. Calc. for $\mathrm{C}_{14} \mathrm{H}_{9} \mathrm{BrN}_{2}$ : C, 58.97; H, 3.18; N, 9.82. Found: C, 58.74; H, 3.35; N, 9.77\%.

\section{2-(4-chlorophenyl)quinoxaline (3b):}

Pale yellow solid; mp: $138{ }^{\circ} \mathrm{C} .{ }^{1} \mathrm{H}$ NMR $\left(\mathrm{CDCl}_{3}\right): \delta 7.53(\mathrm{~d}, J=8.4 \mathrm{~Hz}, 2 \mathrm{H}, \mathrm{ArH}), 7.77$ (bs, 2H, $\mathrm{ArH}), 8.13-8.15$ (m, 4H, ArH), 9.29 (bs, $1 \mathrm{H}, \mathrm{ArH}) \mathrm{ppm} ;{ }^{13} \mathrm{C} \mathrm{NMR}\left(\mathrm{CDCl}_{3}\right): \delta 128.74,129.14$, $129.37,129.58,129.77,130.46,135.13,136.57,141.60,142.23,142.77,150.55$ ppm; FT-IR (KBr): $v$ 2963, 2936, 2834, 1580, 1489, 1452, 1427, 1313, 1093, 832, 755, 699, $548 \mathrm{~cm}^{-1}$. Anal. Calc. for $\mathrm{C}_{14} \mathrm{H}_{9} \mathrm{ClN}_{2}$ : C, 69.86; H, 3.77; N, 11.64. Found: C, 69.66; H, 3.81; N, 11.71\%.

\section{2-(4-methoxyphenyl)quinoxaline (3c):}

Cream solid; mp: $92{ }^{\circ} \mathrm{C} .{ }^{1} \mathrm{H}$ NMR $\left(\mathrm{CDCl}_{3}\right): \delta 3.90\left(\mathrm{~s}, 3 \mathrm{H}, \mathrm{OCH}_{3}\right), 7.08(\mathrm{~d}, J=8.4 \mathrm{~Hz}, 2 \mathrm{H}, \mathrm{ArH}), 7.69-$ 7.78 (m, 2H, ArH), 8.08-8.15 (m, 2H, ArH), 8.18 (d, J = 8.4 Hz, 2H, ArH), 9.30 (s, 1H, ArH) ppm; ${ }^{13} \mathrm{C} \mathrm{NMR}\left(\mathrm{CDCl}_{3}\right): \delta 55.43,114.60,128.98,129.03,129.08,129.22,129.36,130.21,141.14,142.27$, 143.00, 151.40, 161.49 ppm; FT-IR (KBr): v 3056, 2934, 2833, 1606, 1520, 1324, 1250, 1073, 1029 $\mathrm{cm}^{-1}$. Anal. Calc. for $\mathrm{C}_{15} \mathrm{H}_{12} \mathrm{~N}_{2} \mathrm{O}: \mathrm{C}, 76.25 ; \mathrm{H}, 5.12 ; \mathrm{N}, 11.86$. Found: $\mathrm{C}, 76.39 ; \mathrm{H}, 5.01 ; \mathrm{N}, 11.74 \%$.

\section{2-(3,4-dibromophenyl)quinoxaline (3d):}

Orang solid; mp: $115^{\circ} \mathrm{C} .{ }^{1} \mathrm{H}$ NMR $\left(\mathrm{CDCl}_{3}\right): \delta 7.45$ (s, 1H, ArH), $7.66(\mathrm{~d}, J=6.3 \mathrm{~Hz}, 1 \mathrm{H}, \mathrm{ArH}), 7.81$ (bs, 2H, ArH), 8.17 (bs, 2H, ArH), 8.40 (bs, $1 \mathrm{H}, \mathrm{ArH}), 9.31$ (bs, $1 \mathrm{H}, \mathrm{ArH}) \mathrm{ppm} ;{ }^{13} \mathrm{C} \mathrm{NMR}\left(\mathrm{CDCl}_{3}\right): \delta$ $116.30,116.58,123.87,126.03,128.87,129.70,130.27,130.64,130.77,133.25,138.32,142.34$, 142.57, 150.34 ppm; FT-IR (KBr): v 3058, 1566, 1549, 1479, 1311, 1080, 960, 758, $687 \mathrm{~cm}^{-1}$. Anal. Calc. for $\mathrm{C}_{14} \mathrm{H}_{8} \mathrm{Br}_{2} \mathrm{~N}_{2}$ : C, 46.19; H, 2.22; N, 7.70. Found: C, 46.01; H, 2.51; N, 7.62\%. 
2-(3,4-dimethoxyphenyl)quinoxaline (3e):

Yellow solid; mp: $105{ }^{\circ} \mathrm{C} .{ }^{1} \mathrm{H}$ NMR $\left(\mathrm{CDCl}_{3}\right): \delta 4.00\left(\mathrm{~s}, 3 \mathrm{H}, \mathrm{OCH}_{3}\right), 4.08\left(\mathrm{~s}, 3 \mathrm{H}, \mathrm{OCH}_{3}\right), 7.05(\mathrm{~d}, J=$ $8.4 \mathrm{~Hz}, 1 \mathrm{H}, \mathrm{ArH}), 7.74-7.82$ (m, 3H, ArH), 7.89 (s, 1H, ArH), 8.11-8.18 (m, 2H, ArH), 9.33 (s, 1H, ArH) ppm; ${ }^{13} \mathrm{C}$ NMR $\left(\mathrm{CDCl}_{3}\right): \delta 56.04,56.10,110.17,111.15,120.50,129.00,129.19,129.27$, 129.39, 130.30, 141.12, 142.12, 143.00, 149.74, 151.19, 151.26 ppm; FT-IR (KBr): v 3057, 2925, 2836, 1598, 1518, 1461, 1430, 1285, 1250, 1026, $764 \mathrm{~cm}^{-1}$. Anal. Calc. for $\mathrm{C}_{16} \mathrm{H}_{14} \mathrm{~N}_{2} \mathrm{O}_{2}: \mathrm{C}, 72.16 ; \mathrm{H}$, 5.30; N, 10.52. Found: C, 72.02; H, 5.41; N, 10.63\%.

\section{2-(3-bromophenyl)-6-nitroquinoxaline (3f):}

Yellow solid; mp: $226-228{ }^{\circ} \mathrm{C} .{ }^{1} \mathrm{H}$ NMR $\left(\mathrm{CDCl}_{3}\right): \delta 7.60(\mathrm{t}, J=7.8 \mathrm{~Hz}, 1 \mathrm{H}, \mathrm{ArH}), 7.82(\mathrm{~d}, J=8.1 \mathrm{~Hz}$, $1 \mathrm{H}, \mathrm{ArH}), 8.34-8.43$ (m, 2H, ArH), 8.53-8.58 (m, 2H, ArH), 8.92-8.95 (m, 1H, ArH), $9.82(\mathrm{~s}, 1 \mathrm{H}$, $\mathrm{ArH}) \mathrm{ppm} ;{ }^{13} \mathrm{C} \mathrm{NMR}\left(\mathrm{CDCl}_{3}\right): \delta 123.15,123.91,124.51,125.40,125.74,127.53,131.33,131.86$, 134.52, 137.96, 140.79, 144.42, 146.73, 152.57 ppm; FT-IR (KBr): v 3054, 1551, 1524, 1484, 1350, 1304, 1286, 1193, 1076, 795, $691 \mathrm{~cm}^{-1}$. Anal. Calc. for $\mathrm{C}_{14} \mathrm{H}_{8} \mathrm{BrN}_{3} \mathrm{O}_{2}: \mathrm{C}, 50.93 ; \mathrm{H}, 2.44 ; \mathrm{N}, 12.73$. Found: C, 50.77; H, 2.32; N, 12.82\%.

\section{2-(4-chlorophenyl)-6-nitroquinoxaline (3g):}

Cream solid; mp: $261-263{ }^{\circ} \mathrm{C} .{ }^{1} \mathrm{H}$ NMR (DMSO- $\left.d_{6}\right): \delta 7.71(\mathrm{~d}, J=8.4 \mathrm{~Hz}, 2 \mathrm{H}, \mathrm{ArH}), 8.35(\mathrm{~d}, J=8.7$ $\mathrm{Hz}, 1 \mathrm{H}, \operatorname{ArH}), 8.45$ (d, $J=7.8 \mathrm{~Hz}, 2 \mathrm{H}, \operatorname{ArH}), 8.57$ (d, $J=8.1 \mathrm{~Hz}, 1 \mathrm{H}, \operatorname{ArH}), 8.92(\mathrm{~s}, 1 \mathrm{H}, \operatorname{ArH}), 9.81$ (s, 1H, ArH) ppm; FT-IR (KBr): v 3052, 2981, 1622, 1592, 1489, 1435, 1311, 834, $780 \mathrm{~cm}^{-1}$. Owing to extreme insolubility, its ${ }^{13} \mathrm{C}$ NMR spectrum could not be measured in any solvent. Anal. Calc. for $\mathrm{C}_{14} \mathrm{H}_{8} \mathrm{ClN}_{3} \mathrm{O}_{2}$ : C, 58.86; H, 2.82; N, 14.71. Found: C, 58.75; H, 2.78; N, 14.88\%.

\section{2-(4-fluorophenyl)-6-nitroquinoxaline (3h):}

Yellow solid; mp: $234-236{ }^{\circ} \mathrm{C} .{ }^{1} \mathrm{H}$ NMR (DMSO- $\left.d_{6}\right): \delta 7.48(\mathrm{t}, J=8.7 \mathrm{~Hz}, 2 \mathrm{H}, \mathrm{ArH}), 8.33(\mathrm{~d}, J=9$ $\mathrm{Hz}, 1 \mathrm{H}, \mathrm{ArH}), 8.46-8.51$ (m, 3H, ArH), 8.90 (d, $J=2.1 \mathrm{~Hz}, 1 \mathrm{H}, \mathrm{ArH}), 9.79(\mathrm{~s}, 1 \mathrm{H}, \mathrm{ArH}) \mathrm{ppm} ;{ }^{13} \mathrm{C}$ NMR (DMSO-d $d_{6}$ ): $\delta 116.67,116.96,123.53,124.44,125.39,125.52,130.88,131.01,131.13,131.31$, 131.49, 143.84, 146.61, 147.39, 147.64 ppm; FT-IR (KBr): v 3055, 2925, 2853, 1555, 1524, 1350, $1164,838 \mathrm{~cm}^{-1}$. Anal. Calc. for $\mathrm{C}_{14} \mathrm{H}_{8} \mathrm{FN}_{3} \mathrm{O}_{2}: \mathrm{C}, 62.46 ; \mathrm{H}, 3.00 ; \mathrm{N}, 15.61$. Found: C, 62.54; H, 2.98; $\mathrm{N}, 15.48 \%$.

\section{2-(4-nitrophenyl)-6-nitroquinoxaline (3i):}

Cream brown solid; mp: $237-239{ }^{\circ} \mathrm{C} .{ }^{1} \mathrm{H}$ NMR (DMSO- $d_{6}$ ): $\delta$ 8.37-8.44 (m, 3H, ArH), 8.57-8.65 (m, $3 \mathrm{H}, \mathrm{ArH}$ ), 8.93 (bs, $1 \mathrm{H}, \mathrm{ArH}), 9.87$ (s, 1H, ArH) ppm; ${ }^{13} \mathrm{C}$ NMR (DMSO-d $\left._{6}\right): \delta 124.66,125.42$, $129.81,131.40,131.85,140.68,141.47,146.89,147.68,148.22,149.41,152.08$ ppm; FT-IR (KBr): $v$ $3093,2942,1555,1519,1347,854,755,692 \mathrm{~cm}^{-1}$. Anal. Calc. for $\mathrm{C}_{14} \mathrm{H}_{8} \mathrm{~N}_{4} \mathrm{O}_{4}: \mathrm{C}, 56.76 ; \mathrm{H}, 2.72 ; \mathrm{N}$, 18.91. Found: C, 56.66; H, 2.63; N, 18.98\%.

\section{2-([1,1'-biphenyl]-4-yl)-6-nitroquinoxaline (3j):}

Green yellow; mp: $171-174{ }^{\circ} \mathrm{C} .{ }^{1} \mathrm{H}$ NMR (DMSO- $\left.d_{6}\right): \delta$ 7.40-7.49 (m, 3H, ArH), $7.77(\mathrm{~d}, J=6.8 \mathrm{~Hz}$, 2H, ArH), 7.91 (d, $J=7.5 \mathrm{~Hz}, 2 \mathrm{H}, \mathrm{ArH}), 8.32$ (d, $J=9.3 \mathrm{~Hz}, 1 \mathrm{H}, \mathrm{ArH}), 8.48$ (bs, 3H, ArH), 8.89 (s, $1 \mathrm{H}, \mathrm{ArH}), 9.82(\mathrm{~s}, 1 \mathrm{H}, \mathrm{ArH}) ;{ }^{13} \mathrm{C}$ NMR (DMSO- $\left.d_{6}\right): \delta 123.48,124.42,125.56,127.31,127.90$, $128.67,128.89,129.11,129.56,131.31,134.54,139.45,143.15,143.97,147.50,148.45$ ppm; FT-IR $(\mathrm{KBr}): v$ 3055, 2930, 1605, 1547, 1522, 1345, 1191, 1050, 766, $728 \mathrm{~cm}^{-1}$. Anal. Calc. for $\mathrm{C}_{20} \mathrm{H}_{13} \mathrm{~N}_{3} \mathrm{O}_{4}$ : C, 73.38; H, 4.00; N, 12.84. Found: C, 73.25; H, 3.88; N, 12.95\%.

\section{2-phenyl-6/7-methylquinoxaline (3k):}

Cream solid; mp: 89-93 ${ }^{\circ} \mathrm{C}$, isomers ratio (67:33). ${ }^{1} \mathrm{H}$ NMR $\left(\mathrm{CDCl}_{3}\right): \delta 2.62\left(\mathrm{~s}, 3 \mathrm{H}, \mathrm{CH}_{3}\right), 7.55-7.64$ (m, 4H, ArH), 7.95-8.03 (m, 2H, ArH), 8.20 (d, J=6.3 Hz, 2H, ArH), 9.27 (s, 1H, H-3, one isomer), 9.29 (s, $1 \mathrm{H}, \mathrm{H}-3$, other isomer) ppm; ${ }^{13} \mathrm{C} \mathrm{NMR}\left(\mathrm{CDCl}_{3}\right): \delta 21.87,127.43,127.51,127.90,128.46$, 128.58, 129.13, 129.99, 130.08, 131.92, 132.67, 138.31, 142.40 ppm; FT-IR (KBr): v 3055, 2917, 
2854, 1540, 1491, 1307, 1026, 827, 766, $688 \mathrm{~cm}^{-1}$. Anal. Calc. for $\mathrm{C}_{20} \mathrm{H}_{13} \mathrm{~N}_{3} \mathrm{O}_{4}: \mathrm{C}, 81.79 ; \mathrm{H}, 5.49 ; \mathrm{N}$, 12.72. Found: C, $81.63 ; \mathrm{H}, 5.35 ; \mathrm{N}, 12.95 \%$.

\section{2-(3-bromophenyl)-6/7-methylquinoxaline (3l):}

Cream brown solid; mp: 101-104 ${ }^{\circ} \mathrm{C}$, isomers ratio (66:34). ${ }^{1} \mathrm{H}$ NMR $\left(\mathrm{CDCl}_{3}\right): \delta 2.63\left(\mathrm{~s}, 3 \mathrm{H}, \mathrm{CH}_{3}\right)$, $7.44(\mathrm{t}, J=7.8 \mathrm{~Hz}, 1 \mathrm{H}, \operatorname{ArH}), 7.67-7.69(\mathrm{~m}, 2 \mathrm{H}, \operatorname{ArH}), 7.93(\mathrm{~d}, J=7.8 \mathrm{~Hz}, 1 \mathrm{H}, \operatorname{ArH}), 8.05(\mathrm{~d}, J=8.1$ $\mathrm{Hz}, 1 \mathrm{H}, \mathrm{ArH}), 8.08-8.11(\mathrm{~m}, 1 \mathrm{H}, \mathrm{ArH}), 8.38(\mathrm{~s}, 1 \mathrm{H}, \mathrm{ArH}), 9.23(\mathrm{~s}, 1 \mathrm{H}, \mathrm{H}-3$, one isomer $), 9.26(\mathrm{~s}, 1 \mathrm{H}$, $\mathrm{H}-3$, other isomer $) \mathrm{ppm} ;{ }^{13} \mathrm{C} \mathrm{NMR}\left(\mathrm{CDCl}_{3}\right): \delta 21.92,123.45,125.82,125.92,127.76,128.47,129.13$, $130.45,130.54,130.59,138.44,132.95,133.02,141.49,142.45$ ppm; FT-IR (KBr): v 3058, 2913, 1620, 1538, 1439, 1288, 1058, 966, 822, 774, $689 \mathrm{~cm}^{-1}$. Anal. Calc. for $\mathrm{C}_{15} \mathrm{H}_{11} \mathrm{BrN}_{2}: \mathrm{C}, 60.22 ; \mathrm{H}$, 3.71 ; N, 9.36. Found: C, 60.39; H, 3.63; N, 9.17\%.

\section{2-(3-methoxyphenyl)-6/7-methylquinoxaline (3m):}

Cream solid; mp: $84-87{ }^{\circ} \mathrm{C}$, isomers ratio (68:32). ${ }^{1} \mathrm{H}$ NMR $\left(\mathrm{CDCl}_{3}\right): \delta 2.61\left(\mathrm{~s}, 3 \mathrm{H}, \mathrm{CH}_{3}\right), 3.94(\mathrm{~s}, 3 \mathrm{H}$, $\left.\mathrm{OCH}_{3}\right), 7.07$ (d, $\left.J=8.1 \mathrm{~Hz}, 1 \mathrm{H}, \mathrm{ArH}\right), 7.47$ (t, $\left.J=7.8 \mathrm{~Hz}, 1 \mathrm{H}, \mathrm{ArH}\right), 7.72-7.76$ (m, 2H, ArH), 7.88$8.07(\mathrm{~m}, 2 \mathrm{H}, \mathrm{ArH}), 9.25$ (s, 1H, H-3, one isomer), 9.26 (s, 1H, H-3, other isomer) ppm; ${ }^{13} \mathrm{C} \mathrm{NMR}$ $\left(\mathrm{CDCl}_{3}\right): \delta 21.87,55.45,112.50,112.59,116.03,116.12,119.77,119.85,127.88,128.45,128.55$, $129.12,130.11,131.59,132.65,138.29,140.07,140.25,140.70,140.97,142.28,142.45,143.19$, 151.52, 160.28 ppm; FT-IR (KBr): v 2919, 2835, 1607, 1584, 1542, 1507, 1484, 1460, 1041, 829, 784, $625 \mathrm{~cm}^{-1}$. Anal. Calc. for $\mathrm{C}_{16} \mathrm{H}_{14} \mathrm{~N}_{2} \mathrm{O}: \mathrm{C}, 76.78 ; \mathrm{H}, 5.64 ; \mathrm{N}, 11.19$. Found: C, 76.84; H, 5.52; N, $11.03 \%$.

\section{2-(4-bromophenyl)-6/7-methylquinoxaline (3n):}

Cream gray solid: $\mathrm{mp}: 135-137{ }^{\circ} \mathrm{C}$, isomers ratio (66:34). ${ }^{1} \mathrm{H}$ NMR $\left(\mathrm{CDCl}_{3}\right): \delta 2.63\left(\mathrm{~s}, 3 \mathrm{H}, \mathrm{CH}_{3}\right)$, $7.62(\mathrm{~d}, J=8.7 \mathrm{~Hz}, 1 \mathrm{H}, \mathrm{ArH}), 7.70$ (d, $J=8.7 \mathrm{~Hz}, 2 \mathrm{H}, \mathrm{ArH}), 7.93$ (bs, 1H, ArH), 8.03 (d, $J=8.1 \mathrm{~Hz}$, $1 \mathrm{H}, \mathrm{ArH}), 8.09$ (d, $J=8.7 \mathrm{~Hz}, 2 \mathrm{H}, \mathrm{ArH}), 9.25$ (s, 1H, H-3, one isomer), 9.26 (s, 1H, H-3, other isomer) ppm; ${ }^{13} \mathrm{C}$ NMR $\left(\mathrm{CDCl}_{3}\right): \delta 21.80,21.72,127.94,128.10,128.49,128.61,128.93,128.99$, 131.52 , 132.16, 132.29, 132.67, 132.83, 135.76, 139.43, 139.81, 140.67, 140.94, 141.82, 141.93, 142.58, 151.23, 161.49 ppm; FT-IR (KBr): v 3050, 2915, 1621, 1586, 1488, 1072, 833, 823, 777, 492 $\mathrm{cm}^{-1}$. Anal. Calc. for $\mathrm{C}_{15} \mathrm{H}_{11} \mathrm{BrN}_{2}$ : C, 60.22; H, 3.71; N, 9.36. Found: C, 60.32; H, 3.61; N, 9.18\%.

\section{2-(4-chlorophenyl)-6-methylquinoxaline (3o) :}

Yellow solid; mp: $169-172{ }^{\circ} \mathrm{C} .{ }^{1} \mathrm{H}$ NMR $\left(\mathrm{CDCl}_{3}\right): \delta 2.63\left(\mathrm{~s}, 3 \mathrm{H}, \mathrm{CH}_{3}\right), 7.54(\mathrm{~d}, J=8.4 \mathrm{~Hz}, 2 \mathrm{H}, \mathrm{ArH})$, $7.61(\mathrm{~d}, J=8.4 \mathrm{~Hz}, 1 \mathrm{H}, \mathrm{ArH}), 7.94(\mathrm{~s}, 1 \mathrm{H}, \mathrm{ArH}), 8.03$ (d, $J=8.4 \mathrm{~Hz}, 1 \mathrm{H}, \operatorname{ArH}), 9.16$ (d, $J=8.4 \mathrm{~Hz}$, $2 \mathrm{H}, \mathrm{ArH}), 9.24(\mathrm{~s}, 1 \mathrm{H}, \mathrm{ArH}) \mathrm{ppm} ;{ }^{13} \mathrm{C} \mathrm{NMR}\left(\mathrm{CDCl}_{3}\right): \delta 21.89,128.39,128.54,128.72,129.09$, 129.36, 132.20, 135.27, 136.46, 141.17, 141.80, 142.29, 150.52 ppm; FT-IR (KBr): v 3052, 2921, 1489, 1435, 1311, 1092, 834, $780 \mathrm{~cm}^{-1}$. Anal. Calc. for $\mathrm{C}_{15} \mathrm{H}_{11} \mathrm{ClN}_{2}: \mathrm{C}, 70.73 ; \mathrm{H}, 4.35 ; \mathrm{N}, 11.00$. Found: C, 70.66; H, 4.22; N, 11.16\%.

\section{2-(4-fluorophenyl)-6/7-methylquinoxaline (3p):}

Cream solid; mp: $118-121{ }^{\circ} \mathrm{C}$, isomers ratio (65:35). ${ }^{1} \mathrm{H}$ NMR $\left(\mathrm{CDCl}_{3}\right): \delta 2.62\left(\mathrm{~s}, 3 \mathrm{H}, \mathrm{CH}_{3}\right), 7.23-7.28$ (m, 2H, ArH), 7.60 (d, J=8.7 Hz, 1H, ArH), 7.92 (s, 1H, ArH), 8.02 (bd, J=7.5 Hz, 1H, ArH), 8.19 (t, $J=6.3 \mathrm{~Hz}, 2 \mathrm{H}, \mathrm{ArH}), 9.23$ (s, 1H, H-3, one isomer, ArH), 9.25 (s, 1H, H-3, other isomer) ppm; ${ }^{13} \mathrm{C} \mathrm{NMR}\left(\mathrm{CDCl}_{3}\right): \delta 21.90,116.18,116.47,126.88,127.96,128.20,128.99,129.39,129.52,129.64$, 132.53, 133.47, 138.83, 140.96, 141.73, 142.27, 150.74, 162.71, 166.03 ppm; FT-IR (KBr): v 3052, 2921, 1601, 1499, 1225, 838, $721 \mathrm{~cm}^{-1}$. Anal. Calc. for $\mathrm{C}_{15} \mathrm{H}_{11} \mathrm{FN}_{2}: \mathrm{C}, 75.62 ; \mathrm{H}, 4.65 ; \mathrm{N}, 11.76$. Found: C, 75.87; H, 4.54; N, 11.65\%.

\section{2-(4-methoxyphenyl)-6/7-methylquinoxaline (3q):}

Cream solid; mp: $94-96{ }^{\circ} \mathrm{C}$, isomers ratio (60:40). ${ }^{1} \mathrm{H} \mathrm{NMR}\left(\mathrm{CDCl}_{3}\right): \delta 2.60\left(\mathrm{~s}, 3 \mathrm{H}, \mathrm{CH}_{3}\right), 3.90(\mathrm{~s}, 3 \mathrm{H}$, $\left.\mathrm{OCH}_{3}\right), 7.07(\mathrm{~d}, J=8.4 \mathrm{~Hz}, 2 \mathrm{H}, \mathrm{ArH}), 7.52-7.60(\mathrm{~m}, 1 \mathrm{H}, \mathrm{ArH}), 7.86-8.02(\mathrm{~m}, 2 \mathrm{H}, \mathrm{ArH}), 8.14-8.18$ $(\mathrm{m}, 2 \mathrm{H}, \mathrm{ArH}), 9.23(\mathrm{~s}, 1 \mathrm{H}, \mathrm{H}-3$, one isomer $), 9.24$ (s, $1 \mathrm{H}, \mathrm{H}-3$, other isomer) ppm; ${ }^{13} \mathrm{C} \mathrm{NMR}\left(\mathrm{CDCl}_{3}\right)$ : 
$\delta 21.80,55.43,114.55,127.84,128.20,128.50,128.82,128.86,128.93,129.35,131.42,132.54$, 139.60, 140.77, 142.07, 142.27, 142.81, 150.64, 151.29, 161.30, 161.40 ppm; FT-IR (KBr): v 2921, 2852, 1605, 1433, 1325, 1254, 1179, 1027, $831 \mathrm{~cm}^{-1}$. Anal. Calc. for $\mathrm{C}_{16} \mathrm{H}_{14} \mathrm{~N}_{2} \mathrm{O}: \mathrm{C}, 76.78 ; \mathrm{H}, 5.64$; N, 11.19. Found: C, 76.85; H, 5.55; N, 11.25\%.

\section{2-(4-nitrophenyl)-6/7-methylquinoxaline (3r):}

Gray solid; mp: $145-148{ }^{\circ} \mathrm{C}$, isomers ratio (64:36). ${ }^{1} \mathrm{H}$ NMR $\left(\mathrm{CDCl}_{3}\right): \delta 2.63\left(\mathrm{~s}, 3 \mathrm{H}, \mathrm{CH}_{3}\right), 7.65(\mathrm{~d}, J$ $=6.9 \mathrm{~Hz}, 1 \mathrm{H}, \mathrm{ArH}), 7.93(\mathrm{~d}, J=9 \mathrm{~Hz}, 1 \mathrm{H}, \mathrm{ArH}), 8.05(\mathrm{~d}, J=7.8 \mathrm{~Hz}, 1 \mathrm{H}, \operatorname{ArH}), 8.30-8.38(\mathrm{~m}, 4 \mathrm{H}$, $\mathrm{ArH}), 9.30(\mathrm{~s}, 1 \mathrm{H}, \mathrm{H}-3$, one isomer $), 9.32(\mathrm{~s}, 1 \mathrm{H}, \mathrm{H}-3$, other isomer $) \mathrm{ppm} ;{ }^{13} \mathrm{C} \mathrm{NMR}\left(\mathrm{CDCl}_{3}\right): \delta$ $21.91,124.25,128.04,128.13,128.24,128.58,128.73,129.33,133.07,133.25,141.58,141.88$, 142.69 ppm; FT-IR (KBr): v 2922, 1600, 1518, 1490, 1345, 1049, 960, 854, $692 \mathrm{~cm}^{-1}$. Anal. Calc. for $\mathrm{C}_{15} \mathrm{H}_{11} \mathrm{~N}_{3} \mathrm{O}_{2}$ : C, 67.92; H, 4.18; N, 15.84. Found: C, 67.85; H, 4.22; N, 15.92\%.

\section{2-(3,4-dimethoxyphenyl)-6/7-methylquinoxaline (3s):}

Cream brown solid; mp: 97-100 ${ }^{\circ} \mathrm{C}$, isomers ratio (56:44). ${ }^{1} \mathrm{H}$ NMR $\left(\mathrm{CDCl}_{3}\right): \delta 2.61\left(\mathrm{~s}, 3 \mathrm{H}, \mathrm{CH}_{3}\right)$, $3.98\left(\mathrm{~s}, 3 \mathrm{H}, \mathrm{OCH}_{3}\right), 4.06\left(\mathrm{~s}, 3 \mathrm{H}, \mathrm{OCH}_{3}\right), 7.03(\mathrm{~d}, J=8.4 \mathrm{~Hz}, 1 \mathrm{H}, \mathrm{ArH}), 7.54-7.62(\mathrm{~m}, 1 \mathrm{H}, \mathrm{ArH}), 7.72$ (d, $J=7.2 \mathrm{~Hz}, 1 \mathrm{H}, \mathrm{ArH}), 7.86$ (bs, 2H, ArH), 7.95-8.06 (m, 1H, ArH), 9.24 (s, 1H, H-3, one isomer), $9.26\left(\mathrm{~s}, 1 \mathrm{H}, \mathrm{H}-3\right.$, other isomer) $\mathrm{ppm} ;{ }^{13} \mathrm{C} \mathrm{NMR}\left(\mathrm{CDCl}_{3}\right): \delta 21.79,21.86,56.01,56.06,110.02$, $110.10,111.12,120.23,120.36,127.88,128.16,128.51,128.81,129.60,131.46,132.55,139.65$, 139.70, 140.59, 140.79, 141.16, 142.12, 142.88, 149.67, 150.50, 150.93, 151.03, 141.15 ppm; FT-IR (KBr): $v$ 3081, 2934, 2837, 1600, 1519, 1501, 1288, 1249, 1173, $1023 \mathrm{~cm}^{-1}$. Anal. Calc. for $\mathrm{C}_{17} \mathrm{H}_{16} \mathrm{~N}_{2} \mathrm{O}_{2}$ : C, 72.84; H, 5.75; N, 9.99. Found: C, 72.75; H, 5.64; N, 9.78\%.

\section{2-([1,1'-biphenyl]-4-yl)-6/7-methylquinoxaline (3t);}

Cream solid; mp: $84-87^{\circ} \mathrm{C}$, isomers ratio (65:35). ${ }^{1} \mathrm{H}$ NMR $\left(\mathrm{CDCl}_{3}\right): \delta 2.64\left(\mathrm{~s}, 3 \mathrm{H}, \mathrm{CH}_{3}\right), 7.42(\mathrm{~d}, J=$ $6.9 \mathrm{~Hz}, 1 \mathrm{H}, \mathrm{ArH}), 7.50(\mathrm{t}, J=6.3 \mathrm{~Hz}, 2 \mathrm{H}, \mathrm{ArH}), 7.61(\mathrm{~d}, J=8.4 \mathrm{~Hz}, 1 \mathrm{H}, \operatorname{ArH}), 7.69(\mathrm{~d}, J=7.2 \mathrm{~Hz}$, 2H, ArH), 7.80-7.99 (m, 3H, ArH), 8.04 (d, J=7.8 Hz, 1H, ArH), 8.29 (d, J=7.8 Hz, 2H, ArH), 9.32 (s, 1H, H-3, one isomer), $9.34\left(\mathrm{~s}, 1 \mathrm{H}, \mathrm{H}-3\right.$, other isomer) ppm; ${ }^{13} \mathrm{C} \mathrm{NMR}\left(\mathrm{CDCl}_{3}\right): \delta 22.04,126.13$, $127.18,127.46,127.98,128.01,128.13,128.97,132.94,133.92,134.50,140.01,140.58,142.16$, 142.27, 143.55, 151.28 ppm; FT-IR (KBr): v 3029, 2916, 1602, 1534, 1486, 1052, 845, 764, $689 \mathrm{~cm}^{-}$ 1. Anal. Calc. for $\mathrm{C}_{21} \mathrm{H}_{16} \mathrm{~N}_{2}$ : C, 85.11; H, 5.44; N, 9.45. Found: C, 85.30; H, 5.34; N, 9.32\%.

\section{References}

1. Zarranz, B., Jaso, A., Aldana, I., and Monge, A. (2004) Synthesis and anticancer activity evaluation of new 2-alkylcarbonyl and 2-benzoyl-3-trifluoromethyl-quinoxaline 1,4-di-Noxide derivatives, Bioorg. Med. Chem., 12, 3711-3721.

2. Lee, H., Cho, S., Namgoong, K., Jung, J. K., and Yang, S. (2004) Synthesis and in vitro evaluation of 7-dialkylaminomethylbenzo[g]quinoxaline-5,10-diones, Bioorg. Med. Chem. Lett., 14, 1235-1237.

3. Chung, H. J., Jung, O. J., Chae, M. J., Hong, S. Y., Chung, K. H., and Lee, S. K. (2005) Synthesis and biological evaluation of quinoxaline-5,8-diones that inhibit vascular smooth muscle cell proliferation, Bioorg. Med. Chem. Lett., 15, 3380-3384.

4. Amin, K. M., Ismail, M. M. F., Noaman, E., Soliman, D. H., and Ammar, Y. A. (2006) New quinoxaline 1,4-di-N-oxides. Part 1: Hypoxia-selective cytotoxins and anticancer agents derived from quinoxaline 1,4-di-N-oxides, Bioorg. Med. Chem., 14, 6917-6923.

5. Grande, F., Aiello, F., Grazia, O. D., Brizz,i A., Garofalo, A., and Neamati, N. (2007) Synthesis and antitumor activities of a series of novel quinoxalinhydrazides, Bioorg. Med. Chem., 15, 288-294. 
6. Diana, P., Martorana, A., Barraja, P., and Montalbaro, A. (2008) Isoindolo[2,1-a]quinoxaline Derivatives, Novel Potent Antitumor Agents with Dual Inhibition of Tubulin Polymerization and Topoisomerase I, J. Med. Chem., 51, 2387-2399.

7. Tandon, V. K., Yadav, D. B., Maurya, H. K., Chaturvedi, A. K., and Shukla, P. K. (2006) Design, synthesis, and biological evaluation of 1,2,3-trisubstituted-1,4dihydrobenzo[g]quinoxaline-5,10-diones and related compounds as antifungal and antibacterial agents, Bioorg. Med. Chem., 14, 6120-6126.

8. Kotharkar, S. A., and Shinde, D. B. (2006) Synthesis of antimicrobial 2,9,10-trisubstituted-6oxo-7,12-dihydro-chromeno[3,4-b]quinoxalines, Bioorg. Med. Chem. Lett., 16, 6181-6184.

9. Mashevskaya, I. V., Makhmudov, R. R., Aleksandrova, G. A., Golovnira, O. V., Duvalov, A. V., and Maslivets, A. N. (2001) Synthesis and Study of the Antibacterial and Analgesic Activity of 3-Acyl-1,2,4,5-tetrahydro-[1,2-a]quinoxaline-1,2,4-triones, Pharm. Chem. J., 35, 196-198.

10. Vyas, D. A., Chauhan, N. A., and Parikh, A. R. (2007) Synthesis and anti microbial activity of quinoxaline based thiazolidinones and azetidinones, Indian J. Chem., 46B, 1699-1702.

11. Carta, A., Loriga, M., Paglietti, G., Mattana, A., Fiori, P. L., Mollicotti, P., Sechi, L., and Zanetti, S. Synthesis, anti-mycobacterial, anti-trichomonas and anti-candida in vitro activities of 2-substituted-6,7-difluoro-3-methylquinoxaline 1,4-dioxides, Eur. J. Med. Chem., 39, 195203.

12. Seitz, L. E., Suling, W. J., and Reynolds, R. C. (2002) Synthesis and Antimycobacterial Activity of Pyrazine and Quinoxaline Derivatives, J. Med. Chem., 45, 5604-5606.

13. Zarranz, B., Jaso, A., Aldana, I., and Monge, A. (2003) Synthesis and antimycobacterial activity of new quinoxaline-2-carboxamide 1,4-di-N-oxide derivatives, Bioorg. Med. Chem., $11,2149-2156$.

14. Jaso, A., Zarranz, B., Aldana, I., and Monge, A.(2005) Synthesis of New Quinoxaline-2carboxylate 1,4-Dioxide Derivatives as Anti-Mycobacterium tuberculosis Agents, J. Med. Chem., 48, 2019-2025.

15. Burguete, A., Pontiki, E., Litina, D. H., Villar, R., Vicente, E., Solano, B., Ancizu, S., Aldana, I., and Monge, A. (2007) Synthesis and anti-inflammatory/antioxidant activities of some new ring substituted 3-phenyl-1-(1,4-di-N-oxide quinoxalin-2-yl)-2-propen-1-one derivatives and of their 4,5-dihydro-(1H)-pyrazole analogues, Bioorg. Med. Chem. Lett., 17, 6439-6443.

16. Wagle, S., Adhikari, A. V., and Kumari, N. S. (2008) Synthesis of Some New 2-(3-Methyl-7substituted-2-oxoquinoxalinyl)-5-(aryl)-1,3,4-oxadiazoles as Potential Nonsteroidal Antiinflammatory and Analgesic Agents, Indian J. Chem., 47B, 439-448.

17. Jasouri, S., Khalafy, J., Badali, M., and Prager, R. H. (2011) Synthesis of 9H-indeno [1,2-b] pyrazine and $11 \mathrm{H}$-indeno[1,2-b]quinoxaline derivatives in one-step reaction from 2-bromo-4chloro-1-indanone, S. Afri. J. Chem., 64, 105-107.

18. Poursattar Marjani, A., Khalafy, J., and Prager, R. H. (2012) A novel synthesis of imidazo[1,2-a]quinoxalines, Khim. Geterotsikl. Soedin., 48, in press.

19. Riley, H. A., and GraY, A. R. (1943) Organic Syntheses, Collect. Vol. II, p. 509 (Wiley \& Sons: New York, NY). 